\title{
ANALYTIC SETS AS BRANCHED COVERINGS
}

BY

JOHN STUTZ

\begin{abstract}
In this paper we study the relation between the tangent structure of an analytic set $V$ at a point $p$ and the local representation of $V$ as a branched covering. A prototype for our type of result is the fact that one obtains a covering of minimal degree by projecting transverse to the Zariski tangent cone $C_{3}(V, p)$. We show, for instance, that one obtains the smallest possible branch locus for a branched covering if one projects transverse to the cone $C_{4}(V, p)$. This and similar results show that points where the various tangent cones $C_{i}(V, p), i=4,5,6$, have minimal dimension give rise to the simplest branched coverings. This observation leads to the idea of "Puiseux series normalization", generalizing the situation in one dimension. These Puiseux series allow us to strengthen some results of Hironaka and Whitney on the local structure of certain types of singularities.
\end{abstract}

Introduction. In the study of analytic sets one often uses the fact that locally an analytic set can be represented as a branched covering of an open set in $\boldsymbol{C}^{n}$. The classical method is as follows: given $x \in V$ an analytic subset, of pure dimension $n$, of an open set $U \subset C^{m}$, one selects coordinates $\left(x_{i}\right)$ in $C^{m}$ so that $\pi$, the restriction to some neighborhood $N_{x}$ of $x$ in $V$ of the projection $C^{m} \rightarrow C_{x_{1}, \ldots, x_{n}}$, is light, proper, and has image a domain $D$ in $C_{x_{1}, \ldots, x_{n}}$. Here $C_{x_{1}, \ldots, x_{n}}$ is the space spanned by the coordinates $x_{1}, \ldots, x_{m}$. One arranges things so that $V^{n+1}$, the image of $N_{x}$ under the projection $C^{m} \rightarrow C_{x_{1}, \ldots, x_{n+1}}$, is an analytic set of dimension $n$, and further that $\pi_{n+1}$, the restriction to $V^{n+1}$ of the projection $C_{x_{1}, \ldots, x_{n+1}} \rightarrow C_{x_{1} \ldots, x_{n}}$, is light, proper, and has image $D . V^{n+1}$ is a hypersurface and one sees easily that it is defined by the vanishing of $P_{n+1}(x)$, a Weierstrass polynomial in $x_{n+1}$. One shows that the coordinate $x_{n+1}$ can be chosen so that over a "general point" $\pi^{-1}(y)$ and $\pi_{n+1}^{-1}(y)$ have the same number of points.

The triple $\left(N_{x}, \pi, D\right)$ is what we will call a special branched covering. (We will impose some extra technical restrictions, but these are not important here. See $\$ 2$ for an exact definition.) This is a particular case of a branched covering as introduced in [1].

Of prime importance when working with any branched covering is the nature of its branch locus, that is the set of points where the covering map is not locally biholomorphic. In the case above, $\left(N_{x}, \pi, D\right)$, we denote this by $B\left(N_{x}, \pi\right)$. The classical method does not yield a description of this set. All it shows is that

Received by the editors April 30, 1970 and, in revised form, January 31, 1971.

AMS 1969 subject classifications. Primary 3202, 3210, 3244; Secondary 1401, 1418.

Key words and phrases. Branched cover, tangent cones, Puiseux series, normal flatness, saturation.

Copyright (C) 1972, American Mathematical Society 
$B\left(N_{x}, \pi\right) \subset \pi^{-1}(\delta)$, where $\delta$ is the analytic subset of $D$ defined by the vanishing of the discriminant of $P_{n+1}(x) . \S 1$ is devoted to a description of the branch locus as an analytic set.

There are two elementary questions that one may ask about the branch locus of $\left(N_{x}, \pi, D\right)$ which are outside the scope of the classical theory.

I. Let $\operatorname{Sg} V$, the singular locus of $V$, be as defined in $\$ 1$. Can $\pi$ be chosen so that $B\left(N_{x}, \pi\right)=N_{x} \cap \operatorname{Sg} V$ ?

II. Let $\pi^{n+1}: N_{x} \rightarrow V^{n+1}$ be the restriction of the projection $C^{m} \rightarrow C_{x_{1}, \ldots, x_{n+1}}$. Can $\pi^{n+1}$ be chosen so that $\pi^{n+1}\left(B\left(N_{x}, \pi\right)\right)=B\left(V^{n+1}, \pi_{n+1}\right)$ ?

In $\S 2$ we introduce the tangent cones $C_{i}(V, x), i=3,4,5$, defined by Whitney in [2]. The role of $C_{3}(V, x)$ in describing a branched covering is already well known. (See [3] and [4] for example.) We show that for I and II to have affirmative answers, it is sufficient that $\operatorname{dim} C_{4}(V, x)=n$ and $\operatorname{dim} C_{5}(V, x)=n+1$. In $\S 3$ we show that the sets of points where $\operatorname{dim} C_{4}(V, x)>n$ or $\operatorname{dim} C_{5}(V, x)>n+1$ are analytic subsets of $V$, of codimension $\geqq 2$.

In $\$ 4$ we assume that $\operatorname{dim} \operatorname{Sg} V=n-1$, and introduce the following set:

$$
\begin{aligned}
J=\{x \in V: & \operatorname{Sg} V \text { is an } n-1 \text { manifold, } \\
& \left.\operatorname{dim} C_{4}(V, x)=n, \text { and } \operatorname{dim} C_{5}(V, x)=n+1\right\} .
\end{aligned}
$$

We show that if $x \in J$, then near $x, V$ has a Puiseux series normalization (see Propositions 4.2 and 4.5). Using this, in $\$ 5$, we study the structure of the set $C_{3}(V) \mid J=\bigcup_{x \in J} C_{3}(V, x)$. A complete description of its analytic structure is given in Propositions 5.2 and 5.8.

The results of $\S 5$ are closely related to Zariski's theory of equisingularity. (See [7].) In particular the problem of finding converses to our results on questions I and II leads naturally to a generalization of Zariski's discriminant criterion for equisingularity. These results will appear in [14].

In $\S 6$ we use the above to sharpen the results gotten in [3] on "Smooth Attaching" and the existence of "Smooth Wings." As a consequence of this one should be able to simplify Whitney's proof of the existence of "Analytic stratifications satisfying conditions A and B". Indeed the pair $(V-\operatorname{Sg} V, J)$ already satisfy conditions A and $\mathrm{B}$. (Here and beiow see the appropriate section for definitions.)

In the course of $\S 5$ we introduce an auxiliary tangent cone $K_{S}(V, p)$. In $\S 7$ we relate this cone to the "Blow up of $V$ along $S$ ". This allows us to show that $V$ is normally flat along $J$, and so allows us to place the results of $\$ 5$ in a more intrinsic form. One should contrast this result with [10], where it is shown that any stratification satisfying A and B is normally pseudo flat along each stratum. We conclude this final section by showing that near each point $x \in J, V$ has a "semi-analytic fibration". This was proved by Whitney for hypersurfaces in [2]. In [7] Zariski pointed out the close connection of this result with the theory of saturation. We will present a "Lipschitz version" of this result in [14]. 
Some of the results of this are known, or are closely related to known results. (Compare Lemma 3.3 with [12].) We have included them because we felt the proofs given here were of some independent interest.

We will assume that the reader is familiar with the local theory of analytic sets as presented in [1], [5], and [6]. The results of Chapter 7 of [6] will be especially useful. Beyond this we will try to make the paper relatively self-contained at least in the matter of definitions of terms.

Parts of this paper were contained in the author's thesis at Princeton University. The author wishes to take this opportunity to thank his advisor Professor R. C. Gunning for his help and encouragement. He also wishes to thank Professors Henry Laufer and Helmut Röhrl for their assistance. Finally he wishes to thank the referee for a number of improvements in the text, the most important of which was the removal of an incorrect result, and the idea for a part of the proof of Proposition 4.6.

1. General properties of branched covers. Let $\left(X, \mathcal{O}_{X}\right)$ be a pure $n$-dimensional analytic space, $U$ a domain in $C^{m}$, and $f: X \rightarrow U$ a holomorphic map such that $(X, f, U)$ is a branched cover as defined in [1, Chapter III]. Let $x \in X$; we say that $(X, f, U)$ is not branched at $x$ if there are neighborhoods $N_{x}$ and $N_{f(x)}$ of $x$ and $f(x)$ respectively such that $f \mid N_{x}: N_{x} \rightarrow N_{f(x)}$ is biholomorphic. Let $X^{\prime}$ be the set of points where $(X, f, U)$ is not branched.

1.1. Definition. $B(X, f)=X-X^{\prime}$.

$B(X, f)$ is the branch locus of $(X, f, U)$. We wish to show that it is analytic set. We begin by recalling a few facts about the tangent space to an analytic space.

Let $\left(Y, \mathcal{O}_{Y}\right)$ be an analytic space, not necessarily pure dimensional, and $y \in Y$. Define $T(Y, y)$, the tangent space to $Y$ at $y$, as in Chapter 5 of [1].

1.2. Definition. $T(Y)=\bigcup_{y \in Y} T(Y, y)$.

Following [1], if $\varphi: Y \rightarrow Z$ is a holomorphic map, let $\varphi_{*}(y): T(Y, y) \rightarrow T(Z, \varphi(y))$ be its differential. This gives an obvious map $\varphi_{*}: T(Y) \rightarrow T(Z)$. In the case when $Y$ is an analytic subset of a domain $U \subset C^{m}$, and $\varphi$ is the inclusion map, it is shown in [2] that $\varphi_{*}(T(Y))$ is an analytic subset of $T(U)$. This identification will always be assumed in what follows. In general if $y \in Y$ we may select a neighborhood $N_{y}$ of $y$, a neighborhood $U_{0}$ of 0 in $\boldsymbol{C}^{m}$ with $m$ the tangential dimensional of $Y$ at $y$, and a biholomorphic map into $C^{m}, \varphi: N_{y} \rightarrow U_{0}$, with $\varphi(y)=0$ such that $V=\varphi\left(N_{y}\right)$ is an analytic subset of $U_{0}$. It is easy to see that $\varphi_{*}: T\left(N_{y}\right) \rightarrow T(V)$ is 1-1 and onto. Further if $\varphi^{\prime}$ is a second such map, then it follows immediately from the inverse function theorem that any extension of $\varphi \circ \varphi^{\prime-1}$ will be a holomorphic map between neighborhoods of 0 in $C^{m}$. From this it follows that we may give $T(Y)$ the structure of an analytic space by requiring that any such map $\varphi$ be biholomorphic.

Next we must define $\operatorname{Sg} V$. For $k=1, \ldots, \operatorname{dim} Y$ we have the following.

1.3. Definition. $Y^{(k)}=\{y \in Y: Y$ has a branch of dimension $k$ at $y\}$.

It is shown in [1] that $Y^{(k)}$ is an analytic space of pure dimension $k$. Let $S(Y)$ be the set of points near which $Y$ is not a manifold. 
1.4. Definition. $\operatorname{Sg} Y=S(Y) \cup\left(\bigcup_{0 \leqq k<\operatorname{dim} Y} Y^{(k)}\right)$.

Sg $Y$ is what we will call the singular locus of $Y$. It is an analytic subspace of $Y$ of dimension less than $\operatorname{dim} Y$. It is quite easy to see that $\operatorname{Sg}(X) \subset B(X, f)$ for any $(X, f, U)$. In order to describe $B(X, f)-\mathrm{Sg}(X)$ we need a definition and a lemma.

1.5. Definition. Let $V$ be an analytic subset of a domain $U \subset C^{m}$. Let $W$ be a proper analytic subset of $V$ containing $\operatorname{Sg} V$. A set of holomorphic vector fields $\left(v_{j}\right)$ on $U$ will be called a Whitney vector field set for $V$ relative to $W$ if

(a) $v_{j}(y)=0$ for all $j$ if $y \in W$;

(b) $\{v,(y)\}$ spans $T(V, y)$ if $y \in V-W$.

If one has $V$ and $W$ as in 1.5, and $y \in V$ then it follows from Lemma 15.1 in [3] that there is neighborhood $N_{y}$ of $y$ in $C^{m}$ on which there is a Whitney vector field set for $N_{y} \cap V$ relative to $N_{y} \cap W$.

1.6. Lemma. Let $V, U$ and $W$ be as in $1.5, T$ an analytic set of dimension $l$ contained in a domain $S \subset C^{t}$, and $g: V \rightarrow T$ a holomorphic map onto $T$. Suppose that $g^{-1}(\operatorname{Sg} T)$ $\subset W$ and set

$$
W^{\prime}=W \cup\left\{y \in V-W: \operatorname{rank} g_{*}(y)<l\right\} ;
$$

$W^{\prime}$ is an analytic set. If $g$ is proper and $g(W) \neq T$, then $W^{\prime}$ is a proper subset of $V$.

Proof. Let $\left(v_{j}\right)$ be as in 1.5 and $\left(R_{k}\right)$ the set of all $l$-tuples, $\left(v_{j, k}\right)$, of the $v_{j}$. For each $k$ we define a holomorphic, $l \times t$ matrix valued function $Q_{k}$ on $V$ by

$$
Q_{k}(y)=\left[\begin{array}{c}
(\varphi \circ g)_{*}(y)\left(v_{1, k}(y)\right) \\
\vdots \\
(\varphi \circ g)_{*}(y)\left(v_{l, k}(y)\right)
\end{array}\right] .
$$

Here $\varphi: T \rightarrow S$ is the inclusion map. Set $W_{k}=\left\{y \in V: \operatorname{rank} Q_{k}(y)<l\right\}$ and $W^{\prime \prime}$ $=\bigcap_{k} W_{k}$. $W^{\prime \prime}$ is an analytic set. From 1.5(a) it follows that $W \subset W^{\prime \prime}$. From 1.5(b) it follows that $W^{\prime}-W=W^{\prime \prime}-W$ and so $W^{\prime}=W^{\prime \prime}$. Let $\left(V_{j}\right)$ be the set of global branches of $V$ which are not contained in $W$. If $g(W) \neq T$ and $g$ is proper, then $g$ onto implies, for some $j, g\left(V_{j}\right)$ has dimension $l$. For such a $V_{j}$ Propositions 1 and 2 of $\$ 7.2$ of [5] imply that $V_{j} \notin W^{\prime}$. This concludes the proof.

By working locally one easily obtains the following global version of 1.6.

1.7. Corollary. 1.6 continues to hold if we replace $V$ and $W$ by analytic spaces.

Now we come to the main result in this section.

1.8. Let $X$ be a pure $n$-dimensional analytic space and $(X, f, U)$ a branched covering.

(a) $B(X, f)$ is an analytic space of dimension $<n$.

(b) Let $x \in X$. If $\operatorname{dim}_{x} B(X, f)<n-1$ then there is a neighborhood $N_{x}$ of $x$ such that the global branches $\left(X_{j}\right)$ of $N_{x}$ are manifolds and

$$
B(X, f) \cap N_{x}=\bigcup_{\left(j_{1}, j_{2}\right) ; j_{1} \neq j_{2}}\left(X_{j_{1}} \cap X_{j_{2}}\right) .
$$


Proof. $\operatorname{Sg} X \subset B(X, f)$ and it is easy to see that $B(X, f)-\operatorname{Sg} X=\{x \in X-\operatorname{Sg} X$ : rank $\left.f_{*}(x)<n\right\}$. It thus follows from 1.7 that $B(X, f)$ is an analytic set. $f(B(X, f))$ is a negligible analytic set and so is of dimension $<n$. (a) now follows from the equation

$$
\operatorname{dim} f(B(X, f))=\max _{x \in X}\left[\operatorname{dim}_{x} B(X, f)-\operatorname{dim}_{x}\left(f^{-1}(f(x)) \cap B(X, f)\right)\right]
$$

since $f$ is light. Thus (a) is proved.

Suppose that for $x \in X$ we have $\operatorname{dim}_{x} B(X, f)<n-1$. Select $N_{x}$ so that

(a) $\left(N_{x}, f \mid N_{x}, f\left(N_{x}\right)\right)$ is a branched covering;

(b) $\operatorname{dim} B(X, f) \cap N_{x}<n-1$.

We may replace $N_{x}$ by $f^{-1}\left(N_{f(x)}\right)$ where $N_{f(x)}$ is a simply connected open set with $f(x) \in N_{f(x)} \subset f\left(N_{x}\right)$ and so assume that $f\left(N_{x}\right)$ is simply connected. Since $f\left(N_{x}\right)$ is a domain we see that $\left(X_{j}, f \mid X_{j}, f\left(N_{x}\right)\right)$ are all branched coverings which have $\operatorname{dim} f\left(B\left(X_{j}, f \mid X_{j}\right)\right)<n-1$. From 44.45 in [6] it follows that $f\left(N_{x}\right)-f\left(B\left(X_{j}, f \mid X_{j}\right)\right)$ is simply connected and so since $X_{j}$ is globally irreducible that $f \mid X_{j}-B\left(X_{j}, f \mid X_{j}\right)$ is biholomorphic. It now follows from Hartogs' extension theorem that $f \mid X_{j}$ is biholomorphic and so $X_{j}$ is a manifold, and rank $f_{*}(y)=n$ for all $y \in X_{j}$. Using our description of $B(X, f)$ above we see

$$
\operatorname{Sg} X \cap N_{x}=B(X, f) \cap N_{x}=\bigcup_{\left(j_{1}, j_{2}\right): j_{1} \neq j_{2}}\left(X_{j_{1}} \cap X_{j_{2}}\right) .
$$

This completes the proof.

2. Special branched coverings. We begin by fixing our notation. Let $V$ be a pure $n$-dimensional analytic subset of $U$, a domain in $C^{m}$. Let $x_{0} \in V$.

2.1. Definition. $C_{3}\left(V, x_{0}\right)=\left\{v \in C^{m}\right.$ : there are sequences $\left(p_{j}\right) \subset V$ and $\left(a_{j}\right) \subset C$ with $\left(p_{j}\right) \rightarrow x_{0}$ and $\left.\left(a_{j}\left(p_{j}-x_{0}\right)\right) \rightarrow v\right\} . C_{3}\left(V, x_{0}\right)$ is the Zariski tangent cone to $V$ at $x_{0}$.

It is shown in [3] that $C_{3}\left(V, x_{0}\right)$ is a pure $n$-dimensional algebraic set. Let $H$ be an $m-n$ plane in $C^{m}$ with $H \cap C_{3}\left(V, x_{0}\right)=\left(x_{0}\right)$. Select coordinates $\left(x_{i}\right)$ in $C^{m}$ so that $x_{0}$ is the origin and $H=\left\{x \in C^{m}: x_{i}=0\right.$ for $\left.i \leqq n\right\}$, and let $\pi_{H}: V \rightarrow C^{n}$ be the restriction to $V$ of the projection $C^{m} \rightarrow C_{x_{1}, \ldots, x_{n}}$ (this continues the notation of the introduction).

2.2. Proposition. $\pi_{H}^{-1}(0) \cap V$ is discrete.

Proof. $\pi_{H}^{-1}(0) \cap V$ is an analytic set. If it is not discrete there is a sequence $\left(p_{j}\right) \subset \pi_{H}^{-1}(0) \cap V$ with $\left(p_{j}\right) \rightarrow 0$. By passing to a subsequence we may assume that $\left(p_{j} /\left|p_{j}\right|\right) \rightarrow v \neq 0$. Then for any $a \in C$ we have $a\left(p_{j} /\left|p_{j}\right|\right) \rightarrow a v \in H \cap C_{3}(V, 0)$, and so the latter will not be discrete as assumed above. It is shown in [1] that 2.2 implies that there is a neighborhood $L$ of 0 in $C^{m}$ so that if we replace $V$ by $N_{0}=V \cap L$, $\pi_{H}$ by $\pi_{H} \mid V \cap L$ and set $\pi_{H}(V \cap L)=D$ then $\left(N_{0}, \pi_{H}, D\right)$ is a branched covering. In [2] the following is observed.

2.3. $L$ may be taken to be a product $L_{1} \times L_{2}$ with $L_{1}$ open in $C_{x_{1}, \ldots, x_{n}}$ and $L_{2}$ open in $C_{x_{n+1}, \ldots, x_{m}}$ so that

(a) $\pi_{H}\left(N_{0}\right)=L_{1}$, 
(b) if $L_{3} \subset L_{1}$ is an open set with $0 \in L_{3}$ then we may replace $L$ by $L_{3} \times L_{2}$ and all the above continue to hold.

In particular we see that $D$ may be taken to be a polydisk.

2.4. Definition. A branched covering $\left(N_{0}, \pi_{H}, D\right)$ obtained as above will be called a special branched covering.

When we are looking at a fixed covering we will generally suppress the subscript " $H$ " on $\pi$ and " 0 " on $N$. In order to attack question I of the introduction we introduce a second tangent cone.

2.5. Definition. $C_{4}(V, 0)=\left\{v \in C^{m}\right.$ : there are sequences $\left(p_{j}\right)$ and $\left(v_{j}\right)$ with $\left(p_{j}\right) \subset V-\operatorname{Sg} V,\left(p_{j}\right) \rightarrow 0, v_{j} \in T\left(V, p_{j}\right)$, and $\left.\left(v_{j}\right) \rightarrow v\right\}$.

2.6. Proposition. There is a neighborhood $N$ of 0 in $V$ such that $\operatorname{Sg} V \cap N$ $=B(N, \pi)$ if $H \cap C_{4}(V, 0)=(0)$.

Proof. If $B(V, \pi) \neq \operatorname{Sg} V$ in any neighborhood of 0 then we see from our description of $B(V, \pi)$ in $\S 1$ that there is a sequence $\left(p_{j}\right) \subset V-\operatorname{Sg} V$ with $\left(p_{j}\right) \rightarrow 0$ and rank $\pi_{*}\left(p_{j}\right)<n$. Since ker $\pi_{*}\left(p_{j}\right) \subset H$ for all $j$, we can find $v_{j} \in T(V, p)$ so that $\left(v_{j}\right)$ $\rightarrow v$, a nonzero element of $H \cap C_{4}(V, 0)$. This gives a contradiction and so completes the proof.

It is shown in [2] that $C_{3}(V, 0) \subset C_{4}(V, 0)$, and that $C_{4}(V, 0)$ is an algebraic set. Thus to be able to represent $V$, near 0 , as a cover $(N, \pi, D)$ with $B(N, \pi)=\operatorname{Sg} V \cap N$ it is sufficient that $\operatorname{dim} C_{4}(V, 0)=n$. We now go to question II.

Let us assume we have fixed a special branched cover $(N, \pi, D)$ as above. For each integer $i, i=n+1, \ldots, m$, let $\pi^{(i)}: V \rightarrow C_{x_{1}, \ldots, x_{n}, x_{i}}$ be the restriction to $V$ of the projection $C^{m} \rightarrow C_{x_{1}, \ldots, x_{n}, x_{i}}$. It follows from Lemma 9.7 in [3] that we can find $L \subset C^{m}$, a neighborhood of 0 , so that if we replace $N$ by $N \cap L$, and $D$ by $\pi(N \cap D)$ then

2.7. (a) $(N, \pi, D)$ remains a special branched cover.

(b) $\pi^{(i)}(N)=V^{i}$ is an analytic subset of $L^{i}=L \cap C_{x_{1}, \ldots, x_{n}, x_{i}}$ of pure dimension $n$.

(c) If we set $\pi_{i}: V^{i} \rightarrow C_{x_{1}, \ldots, x_{n}}$ equal to the restriction of the projection $C_{x_{1}, \ldots, x_{n}, x_{i}}$ $\rightarrow C_{x_{1}, \ldots, x_{n}}$ then $\pi_{i}\left(V^{i}\right)=D$ and $\left(V^{i}, \pi_{i}, D\right)$ is a special branched covering.

All the assertions except for $\operatorname{dim} V^{i} \equiv n$ are immediate. This follows from the fact that $\left(\pi^{i}\right)^{-1}(0) \subset H \cap \pi^{-1}(0)$ and so $\pi^{i}$ is light near 0 .

2.8. Definition. We will say that $(N, \pi, D)$ has good hypersurface models if, for $i=n+1, \ldots, m$,

(a) $\pi^{i}$ is a homeomorphism;

(b) $\pi^{i}(B(N, \pi))=B\left(V^{i}, \pi_{i}\right)$.

Let $x \in V$. We introduce the tangent cone needed to answer question II.

2.9. Definition. $C_{5}(V, x)=\left\{v \in C^{m}\right.$ : there are sequences $\left(p_{j}\right),\left(q_{j}\right) \subset V$ and $\left(a_{j}\right)$ $\subset C$ so that $\left(p_{j}\right),\left(q_{j}\right) \rightarrow x$ and $\left.\left(a_{j}\left(p_{j}-q_{j}\right)\right) \rightarrow v\right\}$.

For each $i$, set $H^{(i)}=\left\{x \in H: x_{i}=0\right\}$.

2.10. For each $i, 2.8$ (a) and (b) will hold with $N$ replaced by a sufficiently small neighborhood of 0 in $V$ if $H^{i} \cap C_{5}(V, 0)=(0)$. 
Proof. It is immediate that $\pi^{i}$ is continuous and onto. It is open since it is a restriction of the open map $C^{m} \rightarrow C_{x_{1}, \ldots, x_{n}, x_{i}}$. Suppose it is not 1-1 on any neighborhood of 0 . Then we can find sequences $\left(p_{j}\right)$ and $\left(q_{j}\right)$ so that $\pi^{i}\left(p_{j}\right)=\pi^{i}\left(q_{j}\right),\left(p_{j}\right)$, $\left(q_{j}\right) \rightarrow 0$ and $\left(\left(p_{j}-q_{j}\right) /\left|p_{j}-q_{j}\right|\right) \rightarrow v \neq 0$. Since $\left(\pi^{i}\right)^{-1}(x)=\left\{x+y \in C^{m}: x+y \in V\right.$ and $\left.y \in H^{i}\right\}$ for any $x \in V$ we have a contradiction and 2.8(a) is proved. To prove 2.8(b) it suffices to show that $\pi^{i}\left(N_{0}-B(V, \pi)\right)=V^{i}-B\left(V^{i}, \pi_{i}\right)$. Suppose that $x \in V-\operatorname{Sg} V$ with rank $\pi_{*}(x)=n$. Since $\pi_{*}=\pi_{i 。} \circ \pi_{*}^{i}$, rank $\pi_{*}^{i}(x)=n$ and so, since $\pi^{i}$ is a homeomorphism, $\pi^{i}(x) \in V^{i}-\operatorname{Sg} V^{i}$ and rank $\pi_{i_{\bullet}}\left(\pi^{i}(x)\right)=n$. Thus $\pi^{i}(x) \in V^{i}$ $-B\left(V^{i}, \pi_{i}\right)$. Conversely suppose that $x \in V^{i}-\operatorname{Sg} V^{i}$ with rank $\pi_{i \bullet}(x)=n . \pi^{i} \mid N_{0}$ $-B\left(N_{0}, \pi\right)$ is biholomorphic and so by the Riemann extension theorem $\left(\pi^{i}\right)^{-1} \mid V^{i}-\operatorname{Sg} V^{i}$ is holomorphic and so rank $\pi_{*}^{i}\left[\left(\pi^{i}\right)^{-1}\right](x)=n$. Thus rank $\pi_{*}(x)=n$ and this completes the proof.

2.11. Corollary. If $C_{5}(V, 0) \cap H=\{0\}$ then, near $0, V$ is a manifold.

Proof. As above our hypothesis will imply that $\pi_{H}$ is $1-1$. Since $\pi$ is light, $V$ pure dimensional and $D$ nonsingular $\pi$ is open and so a homeomorphism. We see that it is biholomoprhic by using the Riemann extension theorem as above.

2.11 implies that $\operatorname{dim} C_{5}(V, x) \geqq n+1$ if $x \in \mathrm{Sg} V$.

2.12. Proposition. If $\operatorname{dim} C_{5}(V, 0)=n+1$ then we can find coordinates $\left(x_{i}\right)$ such that if $H=\left\{x \in C^{m}: x_{i}=0\right.$ for $\left.i \geqq n\right\}$ then

(a) $H \cap C_{3}(V, 0)=(0)$,

(b) $H^{i} \cap C_{5}(V, 0)=0$ for $i=n+1, \ldots, m$.

Proof. We begin by selecting an $m-(n+1)$ plane $\tilde{H}$ with $\tilde{H} \cap C_{5}(V, 0)=0$. Using Lemma 9.11 in [3] we find an $m-n$ plane $\tilde{H}$ with $\tilde{H} \subset H$ and $C_{3}(V, 0) \cap H=(0)$. Select coordinates so that $H$ is as above and $\tilde{H}=H^{n+1}$.

Let $v \in C^{m}-(0)$ be given, then any $u \in C^{m}-(0)$ can be written uniquely as $u=u_{1}+u_{2}$ where $u_{1}=a v$ and $u_{2} \cdot v=0$. (Here " ." is the inner product relative to the coordinates $\left(x_{i}\right)$.) Define $\pi_{v}: \boldsymbol{C}^{m} \rightarrow \boldsymbol{C}^{m}$ by

$$
\begin{aligned}
\pi_{v}(u) & =u_{1} & & \text { if } u \in C^{m}-(0), \\
& =0 & & \text { if } u=0 .
\end{aligned}
$$

2.13. Definition. For $u, v \in C^{m},|u, v|^{*}=\left|u-\pi_{v}(u)\right| /|u|$.

It is shown in $\S 2$ of [3] that $|,|^{*}$ is symmetric independent of length, and satisfies the "triangle inequality". Since $C_{5}(V, 0)$ is a cone, $C_{5}(V, 0) \cap H^{n+1}=(0)$ implies

$$
\inf _{\tilde{u} \in H^{n+1} ; v \in C_{5}(V, 0)}|\tilde{u}, v|^{*}=\inf _{\tilde{u} \in H^{n+1} ; v \in C_{5}(V, 0) ;|\tilde{u}|=|v|=1}|\tilde{u}, v|^{*}=\delta>0 .
$$

Set $H_{k}=\left\{x \in C^{m}: x_{i}=0\right.$ for $i \leqq n$ and $\left.x_{n+1}+(\delta / 2) x_{k}=0\right\}$ for $k=n+2, \ldots, m$. Let $u=\left(0, \ldots, u_{n+1}, \ldots, u_{m}\right) \in H_{k}$ for some $k, \tilde{u}=\left(0, \ldots, 0, u_{n+2}, \ldots, u_{m}\right) \in H^{n+1}$, then

$$
|u, \tilde{u}|^{*}=\frac{\left|u_{n+1}\right|}{\sum_{i=n+1}^{m}\left|u_{i}\right|}=\frac{\delta}{2} \frac{\left|u_{k}\right|}{\sum_{i=n+1}^{m}\left|u_{i}\right|} \leqq \frac{\delta}{2} \text {. }
$$


This implies that for $v \in C_{5}(V, 0), u \notin H_{k}, \delta \leqq|\tilde{u}, v|^{*} \leqq|u, \tilde{u}|^{*}+|u, v|^{*} \leqq \delta / 2+|u, v|^{*}$ and so $H_{k} \cap C_{5}(V, 0)=(0)$. Select new coordinates $\left(\tilde{x}_{i}\right)$ as follows:

$$
\begin{array}{ll}
\tilde{x}_{i}=x_{i} & \text { for } i \geqq n+1, \\
\tilde{x}_{i}=x_{n+1}+(\delta / 2) x_{i} & \text { for } i \geqq n+2 .
\end{array}
$$

If $F: \boldsymbol{C}^{m} \rightarrow \boldsymbol{C}^{m}$ denotes the map given by this change of coordinates, then $F_{*}(0)\left(H_{k}\right)=H^{k}$ for $k \geqq n+2$, and $F_{*}(0)$ leaves $H$ and $H^{n+1}$ fixed. It is shown in [2] that $F_{*}(0)$ leaves $C_{l}(V, 0)$ fixed for $l=3,5$, so this completes the proof.

Proposition 2.11 gives a sufficient condition for question II to have an affirmative answer. If one wishes to obtain necessary and sufficient conditions for I and II to hold, it seems that in general one will need to consider "relative" tangent cones.

We will illustrate this with an example. Let $H$ be as usual.

2.14. Definition. $C_{4}(V, H, 0)=\left\{v \in C^{m}\right.$ : there are sequences $\left(p_{j}\right)$ and $\left(v_{j}\right)$ with $\left(p_{j}\right) \subset V-\operatorname{Sg} V,\left(p_{j}\right) \rightarrow 0, v_{j} \in T\left(V, p_{j}\right) \cap H$ and $\left.v_{j} \rightarrow v\right\}$.

We get the following extended form of Proposition 2.6.

2.15. Proposition. There is a neighborhood $N$ of 0 in $V$ such that $\left(N, \pi_{H}, D\right)$ is a branched cover with $\operatorname{Sg} V \cap N=B\left(N, \pi_{H}\right)$ if and only if $C_{4}(V, H, 0)=\{0\}$.

The proof is the same as that for Proposition 2.6, so we omit it.

Let us consider $V \subset C^{3}$ given by $V=\left\{\left(x_{1}, x_{2}, x_{3}\right): x_{3}^{2}=x_{1} x_{2}^{2}\right\}$. One sees easily that $\operatorname{Sg} V=C_{x_{1}}$. Because $V$ is a hypersurface one sees that, for $x \in V-\operatorname{Sg} V$,

$$
T(V, x)=\left\{v \in C^{3}:\left(v_{1}, v_{2}, v_{3}\right) \cdot\left(-x_{2}^{2},-2 x_{1} x_{2}, 2 x_{3}\right)=0\right\} .
$$

In this example $H$ will be a line, i.e. $H=\left\{a u \in C^{m}: a \in C\right\}$ for some fixed $u \in C^{3}$. We will show that $C_{4}(V, H, 0) \neq(0)$ for all $H$, and so that $V$ cannot be represented as a special branched covering with branch locus a line. This example is of some importance in the theory of equisaturation (see [7]).

We consider two cases. Write $u=\left(u_{1}, u_{2}, u_{3}\right)$ and assume $u_{1} \neq 0$. For $\left(x_{1}, x_{2}\right)$ $\in \boldsymbol{C}_{x_{1}, x_{2}}-\left(\boldsymbol{C}_{x_{1}} \cup \boldsymbol{C}_{x_{2}}\right)$ we have $\left(x_{1}, x_{2}, x_{1}^{1 / 2} x_{2}\right) \in V$ and so

$$
u \in T\left(V,\left(x_{1}, x_{2}, x_{1}^{1 / 2} x_{2}\right)\right)
$$

if $-x_{2}^{2} u_{1}-2 x_{1} x_{2} u_{2}+x_{1}^{1 / 2} x_{2} u_{3}=0$. If we begin with $x_{1} \in C_{x_{1}}-(0)$ satisfying $-2 x_{1} u_{2}$ $+x_{1}^{1 / 2} u_{3} \neq 0$ we can find $x_{2} \in C_{x_{2}}-(0)$ so that the above hold. From this we see that there is a sequence $\left(p_{j}\right) \subset V-\operatorname{Sg} V$ with $\left(p_{j}\right) \rightarrow 0$ and $u \in T\left(V, p_{j}\right)$ and so $u \in C_{4}(V, H, 0)$. If $u_{1}=0$ then one sees that $u \in T\left(V,\left(0, x_{2}, 0\right)\right)$. This completes our proof.

3. Dimension theorems. Let $V$ be as in $\S 2$. We wish to show that $\{x \in \operatorname{Sg} V$ : $\left.\operatorname{dim} C_{4}(V, x)>n\right\}$ and $\left\{x \in \operatorname{Sg} V: \operatorname{dim} C_{5}(V, x)>n+1\right\}$ are analytic sets of dimension $\leqq n-2$. We begin with two technical lemmas.

3.1. Definition. Let $W$ be an analytic set, $X$ an analytic subset of $W \times C^{l}$, and $g: W \times C^{l} \rightarrow W$ the projection map. We say that $X$ is a holomorphic family of cones over $W$ if 
(a) $\operatorname{dim} g^{-1}(x) \cap X>0$ for all $x \in W$,

(b) if $(x, y) \in X$ then $(x, a y) \in X$ for any $a \in C$.

3.2. Proposition. Let $P^{l-1}$ be the projective $l-1$ space and $f: W \times C^{l}-(0) \rightarrow W$ $\times \boldsymbol{P}^{l-1}$ be the identity across the usual map $C^{l}-(0) \rightarrow \boldsymbol{P}^{l-1}$. Suppose that $X$ satisfies 3.1(b), then $f(X-W \times(0))$ is an analytic subset of $W \times \boldsymbol{P}^{l-1}$.

Proof. Let $\operatorname{dim} X=N$ and define $\left(X^{(k)}\right), k=0, \ldots, N$, as in 1.3. From 3.1 we see that $X^{(0)}$ is empty. Suppose that $(x, y) \in X^{(k)}$ for $k \neq 0$, and let $Y$ be a branch of $X$ through $(x, y)$ of dimension $k$ containing $(x, y)$. For $a \in C, Y_{a}=\left\{\left(x^{\prime}, a y^{\prime}\right):\left(x^{\prime}, y^{\prime}\right)\right.$ $\in Y\}$ is a $k$-dimensional branch of $X$ containing $(x, a y)$. Thus we may assume that $X$ is pure $N$-dimensional.

It follows from [5, Proposition 3, p. 131] that each $x \in X-W \times(0)$ is contained in an open set $N_{x}$ of $W \times C^{l}-(0)$ such that $f\left(N_{x}\right)$ is a neighborhood of $f(x)$ and $f\left(N_{x} \cap X\right)$ is an analytic subset of $f\left(N_{x}\right)$. The problem is to "put together" these local results.

Let $\tilde{g}: W \times \boldsymbol{P}^{l-1} \rightarrow W$ be the projection. Let $T \subset W$ be an open set with compact closure $\bar{T}$. It is sufficient to show that $f(X-W \times(0)) \cap \tilde{g}^{-1}(T)$ is an analytic subset of $\tilde{g}^{-1}(T)$. Let $\widetilde{E}=\left\{z \in C^{m}:|z|=1\right\}$ and $E=W \times \widetilde{E}$. Since $\bar{T}$ is compact we can find a finite set $\left(x_{i}\right) \subset X-W \times(0)$, and $\left(N_{x_{i}}\right)$ as above, so that $\left(N_{x_{i}}\right)$ cover $g^{-1}(T)$ $\cap(X \cap E)$. Now from 3.1(b), we have

$$
f(X-W \times(0)) \cap \tilde{g}^{-1}(T)=f\left(\bigcup N_{x_{l}} \cap X\right) \cap \tilde{g}^{-1}(T)=f(E \cap X) \cap \tilde{g}^{-1}(T) .
$$

Since $N_{x_{i}}$ is finite, $f\left(\cup N_{x_{i}} \cap X\right) \cap \tilde{g}^{-1}(T)$ is an analytic subset of $f\left(\cup N_{x_{i}}\right)$ $\cap \tilde{g}^{-1}(T)$. To see that it is an analytic subset of $\tilde{g}^{-1}(T)$ we must see that it is closed there. $f(E \cap X) \cap \tilde{g}^{-1}(T)$ is easily seen to be closed so this completes the proof.

REMARK. If 3.1(a) also holds then, for all $x \in W$,

$$
\left(\operatorname{dim} \tilde{g}^{-1}(x)\right)+1=\operatorname{dim}\left(g^{-1}(x)-(x \times 0)\right)=\operatorname{dim} g^{-1}(x) \text {. }
$$

3.3. LEMMA. Let $X$ be an analytic space of dimension $r, Y$ an analytic set of dimension $s$ and $h: X \rightarrow Y$ a proper holomorphic map. Suppose that $r \geqq s>0$ and set $A=\left\{y \in Y: \operatorname{dim} h^{-1}(y)>r-s\right\}$. A is an analytic subset of $Y$ of dimension $\leqq s-1$.

Proof. Let $\left(Y_{j}\right)$ be the global irreducible branches of $Y .\left(Y_{j}\right)$ is a locally finite set and $h \mid h^{-1}\left(Y_{j}\right)$ is still proper, so it suffices to prove 3.3 with $Y$ globally irreducible.

Let $\left(X^{(k)}\right)$ be as in 1.3 and let $\left(X_{j}\right)$ be the global branches of $X^{(r)}$. If $\operatorname{dim} h\left(X_{j}\right)<s$ then since

$$
\min _{y \in h\left(X_{j}\right)} \operatorname{dim} h^{-1}(y)=\operatorname{dim} X_{j}-\operatorname{dim} h\left(X_{j}\right)>r-s
$$

we have $h\left(X_{j}\right) \subset A$. If $\operatorname{dim} h\left(X_{j}\right)=s$ then $h\left(X_{j}\right)=Y$ and so we may apply Corollary 1.7 with $W_{j}=\operatorname{Sg} X_{j} \cup h^{-1}(\operatorname{Sg} Y)$. The $W_{j}^{\prime}$ we obtain will be a proper subset of $X_{j}$. From the implicit function theorem we see that if $x \in X_{j}-W_{j}^{\prime}$ then

$$
\operatorname{dim}_{x} h^{-1}(h(x))=r-s .
$$


Let $B=\bigcup_{\left\{X_{j}: \operatorname{dim} h\left(X_{j}\right)<s\right\}} X_{j}$ and $C=\left(\bigcup_{k<r} X^{(k)}\right) \cup\left(\bigcup_{j \in D} W_{j}^{\prime}\right)$ where

$$
D=\left\{X_{j}: \operatorname{dim} h\left(X_{j}\right)=s\right\} .
$$

Then

3.4. $A=B \cup\left\{y \in Y: \operatorname{dim}\left(h^{-1}(y) \cap C\right)>r-s\right\}$.

Define $\left(C^{(k)}\right)$ as usual and assume $C^{(r-1)}$ is not empty. Let $\left(C_{j}\right)$ be its global branches. Since $h\left(C_{j}\right)$ will be globally irreducible, if $\operatorname{dim} h\left(C_{j}\right) \geqq s-1$ we may argue as we did for $X_{j} \in D$ and so replace $C_{j}$, in 3.4 , by a proper analytic subset. If $\operatorname{dim} h\left(C_{j}\right)<s-1$ we may argue as we $\operatorname{did}$ for $X_{j} \subset B$ that $C_{j} \subset A$. Thus in 3.4 we may enlarge $B$ and replace $C$ by an analytic set of dimension $<r-1$. Continuing in this way we will reach a point where $\operatorname{dim} C<r-s$. Then the second set in 3.4 will be empty and we will be done.

Let $V, U$ be as in $\S 2$, then for $i=3,4,5$,

3.5. Definition. (a) $C_{i}(V)=\left\{(x, v) \in U \times C^{m}: x \in V\right.$ and $\left.v \in C_{i}(V, x)\right\}$.

(b) For $S \subset V, C_{i}(V) \mid S=C_{i}(V) \cap S \times C^{m}$.

It is shown in [2] that $C_{i}(V), i=4,5$, are analytic subsets of $U \times C^{m}$. In order to apply the preceding lemmas to $C_{i}(V) \mid \operatorname{Sg} V, i=4,5$, we must compute their dimensions. Let $T(V)$ be as in 1.2, and let $T$ equal the closure of $T(V) \cap(V-\mathrm{Sg} V) \times C^{m}$ in $V \times C^{m}$ intersected with $\mathrm{Sg} V \times C^{m}$. It is shown in [2] that $T=C_{4}(V) \mid \mathrm{Sg} V$, and so it follows from Lemma 2.15 in [2] that $\operatorname{dim} C_{4}(V) \mid \operatorname{Sg} V \leqq 2 n-1$. It is also shown in [2] that $C_{3}(V, x) \subset C_{4}(V, x)$ and $\operatorname{dim} C_{3}(V, x)=n$. The map

$$
g: C_{4}(V) \mid \mathrm{Sg} V \rightarrow \operatorname{Sg} V
$$

induced by projection is onto, so from [6] we see that $\operatorname{dim} C_{4}(V) \mid \operatorname{Sg} V=2 n-1$ if $\operatorname{dim} \operatorname{Sg} V=n-1$.

Let $V^{*}=V \times V \times C^{m}$. For each pair of integers $(i, j), i \neq j, 1 \leqq i, j \leqq m$, we define $\alpha_{i, j}: C^{m} \times C^{m} \times C^{m} \rightarrow C$ by

$$
\alpha_{i, j}(p, q, v)=\operatorname{det}\left(\begin{array}{cc}
p_{i}-q_{i} & p_{j}-q_{j} \\
v_{i} & v_{j}
\end{array}\right) .
$$

The entries in the matrix are the components of $p-q$ and $v$. The vanishing of $\left(\alpha_{i, j}\right)$ defines an analytic set $B$; let $B^{*}=B \cap V^{*}$ and $\Delta$ the diagonal in $V \times V$. We have a map $h: B^{*} \rightarrow \Delta$ induced by projection. As observed in [2], $h \mid B^{*}-h^{-1}(\Delta)$ $\rightarrow V \times V-\Delta$ is onto and has 1-dimensional fibers, and so $\operatorname{dim}\left(B^{*}-h^{-1}(\Delta)\right)$ $=2 n+1$. Let $T^{\prime}$ be the closure of $B^{*}-h^{-1}(\Delta)$ in $V^{*}$ intersected with $h^{-1}(\Delta)$. It is shown in [2] that $T^{\prime}$ is biholomorphically equivalent to $C_{5}(V)$ so $\operatorname{dim} C_{5}(V) \mid \operatorname{Sg} V$ $\leqq 2 n$. From Corollary 2.11 we see that $\operatorname{dim} C_{5}(V, x)>n$ and so as above $\operatorname{dim} C_{5}(V) \mid \operatorname{Sg} V=2 n$ again assuming $\operatorname{dim} \operatorname{Sg} V=n-1$.

Observing that the $C_{i}(V) \mid \mathrm{Sg} V$ satisfy 3.1 with $g$ in each case the projection onto $\operatorname{Sg} V$, we have

3.6. Proposition. $\left\{x \in \operatorname{Sg} V: \operatorname{dim} C_{4}(V, x)>n\right\}$ and $\left\{x \in \operatorname{Sg} V: \operatorname{dim} C_{5}(V, x)>\right.$ $n+1\}$ are analytic sets of dimension $\leqq n-2$. 
Proof. If $\operatorname{dim} \mathrm{Sg} V<n-1$ then the proposition is immediate; if $\operatorname{dim} \mathrm{Sg} V=n-1$ then it follows from 3.2 and the remarks following it together with 3.3.

4. A result on normalization. Let $V, U$ be as usual and assume that $\operatorname{dim} \operatorname{Sg} V$ $=n-1$. Set $\operatorname{Sg}^{2} V=\operatorname{Sg}(\operatorname{Sg} V)$. For a fixed $x_{0} \in \operatorname{Sg} V-\operatorname{Sg}^{2} V$, let $N$ be a neighborhood of $x_{0}$ such that $N \cap \mathrm{Sg}^{2} V$ is empty and let $\left(V_{j}\right)$ be the global branches of $N$.

4.1. Definition. $V_{j}$ will be said to have a Puiseux series normalization if there is an $n$-disk $\tilde{D} \subset C^{n}$, and a holomorphic $\operatorname{map} f_{j}: \tilde{D} \rightarrow V_{j}$ such that

(a) $f$ is a homeomorphism,

(b) we can find coordinates $\left(x_{i}\right),\left(y_{i}\right)$ in $C^{n}$ and $C^{m}$ with $x_{0}=0$ and $f_{j}(0)=0$ so that $f_{j}$ can be given in the form

$$
f_{j}(x)=\left(x_{1}, \ldots, x_{n-1}, x_{n}^{p_{j}}, f_{n+1, j}(x), \ldots, f_{m, j}(x)\right) .
$$

We will say that $V$ has a Puiseux series normalization at $x_{0}$ if 4.1 holds for each of $V_{j}$, using the same $\tilde{D}$ and the same coordinates.

4.2 Proposition. If $x_{0} \in \operatorname{Sg} V-\operatorname{Sg}^{2} V$ and $\operatorname{dim} C_{4}\left(V, x_{0}\right)=n$ then $V$ has a Puiseux series normalization at $x_{0}$.

Proof. Let $H$ be an $m-n$ plane through $x_{0}$ and transversal to $C_{4}\left(V, x_{0}\right)$. $T\left(\operatorname{Sg} V, x_{0}\right)=C_{3}\left(\operatorname{Sg} V, x_{0}\right) \subset C_{4}\left(V, x_{0}\right)$ so we can take coordinates $\left(x_{i}\right)$ such that

4.3. (a) $H=\left\{x \in C^{m}: x_{i}=0\right.$ for $\left.i \leqq n\right\}$,

(b) $\operatorname{Sg} V=\left\{x \in U: x_{i}=0\right.$ for $\left.i \geqq n\right\}$.

As in 2.6 we can find a neighborhood $N$ of $x_{0}$ so that if $\pi=\pi_{H}$ and $D=\pi(N)$ then $(N, \pi, D)$ is a special branched cover with $B(N, \pi)=\operatorname{Sg} V \cap N$.

Let $\left(V_{j}\right)$ be the global branches of $N$. Since $V_{j} \notin B(N, \pi)$ for any $j$, we have $\pi\left(V_{j}\right)=D$ and so $\left(V_{j}, \pi \mid V_{j}, D\right)$ is a special branched covering. Let $D^{\prime}=\pi\left(\operatorname{Sg} N_{x_{0}}\right)$ then $\pi \mid V_{j}-\pi^{-1}\left(D^{\prime}\right)$ is a connected $p_{j}$-to-1 covering of $D-D^{\prime}$ for some integer $p_{j}$. Let $\tilde{D}$ be a second copy of $D$ and define $h_{j}: \tilde{D} \rightarrow D$ by $h_{j}(x)=\left(x_{1}, \ldots, x_{n-1}, x_{n}^{p_{j}}\right)$. $h_{j} \mid \tilde{D}-h_{j}^{-1}\left(D^{\prime}\right)$ is also a connected $p_{j}$-to-1 covering of $D-D^{\prime}$ so there is a biholomorphic map $f_{j}^{\prime}: \tilde{D}-h_{j}^{-1}\left(D^{\prime}\right) \rightarrow V_{j}-\pi^{-1}\left(D^{\prime}\right)$ with $h_{j} \mid \tilde{D}-h_{j}^{-1}\left(D^{\prime}\right)=\pi \cdot f_{j}^{\prime}$. Such a homeomorphism exists since the fundamental group of $D-D^{\prime}$ is the integers: It is holomorphic since $h_{j} \mid \tilde{D}-h_{j}^{-1}\left(D^{\prime}\right)$ and $\pi \mid V_{j}-\pi^{-1}\left(D^{\prime}\right)$ are locally biholomorphic. By the Riemann extension theorem $f_{j}^{\prime}$ extends to a map $f_{j}: \widetilde{D} \rightarrow V_{j}$ with $h_{j}=\pi \cdot f_{j}$. From the definition of $h_{j}$ we see that 4.1(b) holds and so that $f_{j}$ is 1-1. Since $V_{j}$ $-\pi^{-1}\left(D^{\prime}\right)$ is dense in $V_{j}$, it is also onto. It is easy to see that $\tilde{D}$ being locally irreducible implies $V_{j}$ locally irreducible and so from [6] we see that $f_{j}$ is open. Thus 4.1(a) holds.

There is a fundamental distinction to be made between the components $\left(V_{j}\right)$.

4.4. Definition. We say that $V_{j}$ gives essential branching if $\operatorname{dim}\left(V_{j} \cap \operatorname{Sg} V\right)$ $=n-1$. If $\operatorname{dim}\left(V_{j} \cap \mathrm{Sg} V\right)<n-1$ we say it gives fake branching.

We will refer to the $V_{j}$ as essential or fake, depending on the type of branching they give. Since $B\left(V_{j}, \pi \mid V_{j}\right) \subset V_{j} \cap \operatorname{Sg} V$ it follows as in 1.8(b) that if $V_{j}$ is fake then $V_{j}$ is nonsingular and $\pi \mid V_{j}$ is biholomorphic. 
4.5. Proposition. If $(N, \pi, D)$ has good hypersurface models then none of the $V$, are fake.

Proof. Let $\pi^{n+1}: N \rightarrow V^{n+1}$ be as in 2.8 and set $\left(V_{j}^{n+1}\right)=\left(\pi^{n+1}\left(V_{j}\right)\right)$. Fix one $V_{j_{0}}$. For any other $V_{j}, x_{0} \in V_{j} \cap V_{j_{0}}$ so $V_{j}^{n+1} \cap V_{j_{0}}^{n+1}$ is not empty. $V_{j}^{n+1}$ is a hypersurface and $V_{j_{0}}^{n+1}$ is globally irreducible, so since $\pi^{n+1}$ is a homeomorphism $\operatorname{dim} V_{j} \cap V_{j_{0}}=\operatorname{dim} V_{j}^{n+1} \cap V_{j_{0}}^{n+1}=n-1$. Since $V_{j} \cap V_{j_{0}} \subset \operatorname{Sg} V \cap N$ we have $\operatorname{dim}\left(V_{j} \cap \operatorname{Sg} V \cap N\right)=\operatorname{dim}\left(V_{j_{0}} \cap \operatorname{Sg} N_{x_{0}}\right)=n-1$. This completes our proof.

Our notion of fake branching does not correspond exactly to the intuitive idea. What we want is to consider as essential all branches which "behave like" nonmanifold branches. For example

4.6. Proposition. If $V_{j}$ is essential, then if we write $f_{i j}=\sum_{k=0}^{\infty} f_{i, j}^{k}\left(x_{1}, \ldots, x_{n-1}\right) x_{n}^{k}$ we will have $f_{i, j}^{k} \equiv 0$ for all $i, j$ when $k<p_{j}$.

Proof. Fix one $V_{j}$. If $V_{j}$ is essential then $\pi^{-1}\left(D^{\prime}\right) \cap V_{j}=\operatorname{Sg} V \cap N=\left\{x \in C^{m}\right.$ : $x_{i}=0$ for $\left.i \geqq n\right\}$ and so $f_{i, j}^{0} \equiv 0$. Suppose that for some $i, k$ with $0<k<p_{j}$ we have $f_{i, j}^{k}$ not identically zero. From the pairs $i, k$, choose $i_{0}, k_{0}$ so that $k_{0}$ is as small as possible. Assume first that $f_{i_{0}, j}^{k_{0}}(0) \neq 0$. Let $P: V_{j} \rightarrow C_{x_{1}, \ldots, x_{n-1}, x_{i_{0}}}$ be the restriction of the coordinate projection. $P^{-1}(0)=0$ so we may select a neighborhood $V_{j, 0}$ of 0 in $V_{j}$ such that if $D_{0}=P\left(V_{j, 0}\right)$ then $\left(V_{j, 0}, P \mid V_{j, 0}, D_{0}\right)$ is a branched covering. For $V_{j, 0}$ small, $f_{i_{0}, j}^{k_{0}}(0) \neq 0$ will imply that $P \mid\left(V_{j, 0}-\mathrm{Sg} V\right)$ is a topological covering map of degree $k_{0}$. However, the original covering $(V, \pi, D)$, used to define the Puiseux series $f_{j}$, was itself defined by projecting along an $n$-plane transverse to $C_{3}(V, 0)$. From Theorem 6.3 in [4] we see that a covering of degree $k_{0}$ is impossible and so $f_{i_{0}, j}^{k_{0}}$ must be a nonunit. In fact, all the terms $f_{i, j}^{k_{0}}$ must be nonunits if they are not identically zero. Now to each vector $a=\left(a_{n}, \ldots, a_{m}\right) \in C^{m-n+1}$ we associate a projection $\pi_{a}: V_{j} \rightarrow C^{n}$ by restricting the map $\left(x_{1}, \ldots, x_{m}\right) \mapsto\left(x_{1}, \ldots, x_{n-1}\right.$, $\left.\sum_{i=n}^{m} a_{i} x_{i}\right)$ to $V_{j}$. Suppose that, for some choice of $a, \pi_{a}$ restricted to a neighborhood $V_{j, 0}$ of 0 in $V_{j}$ defines a branched covering. Then by examining the Jacobian of $\pi_{a} \circ f_{j}$ one sees that $p \in\left(V_{j, 0}-\operatorname{Sg} V\right) \cap B\left(V_{j, 0}, \pi_{a} \mid V_{j, 0}\right)$ if and only if

$$
g\left(f_{j}^{-1}(p)\right)=\frac{\partial}{\partial x_{n}}\left(a_{n} x_{n}^{p_{j}}+\sum_{i=n+1}^{m} a_{i} f_{i, j}\right)\left(f_{j}^{-1}(p)\right)=0 .
$$

Thus $B\left(V_{j, 0}, \pi_{a} \mid V_{j, 0}\right)=\operatorname{Sg} V$ near 0 if and only if $g$ is of the form $g=u x_{n}^{t}$ with $u(0) \neq 0$. Notice that if we expand $g\left(x_{1}, \ldots, x_{n}\right)$ as a power series in $x_{n}$, say $g(x)$ $=\sum_{l=0}^{\infty} g^{l}\left(x_{1} \cdots x_{n-1}\right) x_{n}^{l}$, then the first nonzero coefficient function possible is $g^{k_{0}-1}=k_{0} \sum_{i=m+1}^{n} a_{i} f_{i, j}^{k_{0}}$. By considering the relation required on the initial terms of power series expansions of the $f_{i, j}^{k_{0}}$, one finds that the set of $a \in C^{m-n+1}$ for which $g^{k_{0}-1}$ is not identically zero contains the complement of a proper subspace. However, note that if $g^{k_{0}-1}$ is not identically zero, then, since it is also not a unit, we cannot write $g=u x_{n}^{t}$ as above. Thus among the $a \in C^{m-n+1}$ which define coverings via the maps $\pi_{a}$, those for which $B\left(V_{j, 0}, \pi_{a} \mid V_{j, 0}\right)=\operatorname{Sg} V$ near 0 are contained in a proper subspace of $C^{m-n+1}$. From Proposition 2.5 we see that this is impossible 
since $\operatorname{dim} C_{4}(V, 0)=n$. Thus the assumption of the existence of a nonzero term $f_{i, j}^{k}$ with $0<k<p_{j}$ leads to a contradiction and our proof is complete.

The term "Puiseux series" as employed above is due to Kimura in [8]. 4.2 proves the existence of Puiseux series in all the cases claimed in [8] and so closes the gap in [8] pointed out in [9]. Kimura's criterion for normality follows immediately from our more geometric definition of $f_{j}$.

Finally we note that our conditions on $C_{4}(V, x)$ and $C_{5}(V, x)$ are not necessary. We could obtain Propositions 4.2 and 4.6 by beginning with the assumption that $V$ can be represented as a special branched cover with $B(V, \pi)=\left\{x \in U: x_{i}=0\right.$ for $i \geqq n\}$ all of whose branches are essential.

5. Continuity of $C_{3}(V, y)$ along $J$. Let $x_{0} \in J$. We take $N$ as in the preceding section, and continue the notations used there.

In particular coordinates will be taken so that 4.3 continues to hold. Proceeding as in 2.12 we may modify the $\left(x_{i}\right)$ so that $(N, \pi, D)$ has good hyperplane models. Finally we may assume that $N \cap \operatorname{Sg} V \subset J$. Set $S=N \cap \operatorname{Sg} V$, and for each $y \in S$ let $T_{y}$ be the $m-n+1$ plane orthogonal to $S$ at $y$.

5.1. Definition. (a) $V_{y}^{\perp}=T_{y} \cap N$.

(b) $V_{y, j}^{\perp}=T_{y} \cap V_{j}$.

5.2. Proposition. $C_{3}\left(V_{j}, y\right)=C_{3}\left(V_{j, y}^{\perp}, y\right) \times C_{3}(S, y)$ for all $y \in S$ and all $j$.

Proof. We will show that $C_{3}\left(V_{j}, y\right) \subset C_{3}\left(V_{j, y}^{\perp}, y\right) \times C_{3}(S, y)$. Assuming this we note that

$$
V_{j, y}^{\perp}=f_{j}\left(\left\{x \in \tilde{D}: x_{i}=y_{i} \text { for } i=1, \ldots, n-1\right\}\right)
$$

and so is an irreducible 1-dimensional analytic set. Thus by Lemma 11.2 in [3], $C_{3}\left(V_{j, y}^{1}, y\right)$ is a complex line, and so $C_{3}\left(V_{j, y}^{1}, y\right) \times C_{3}(S, y)$ is an $n$-plane. Since $\operatorname{dim} C_{3}\left(V_{j}, y\right)=n$, the inclusion implies equality.

Let $\left(p_{k}\right) \subset V_{j}$ and $\left(a_{k}\right) \subset C$ be sequences with $\left(p_{k}\right) \rightarrow 0$ and $\left(a_{k} p_{k}\right) \rightarrow v$. We may assume that $v \notin C_{3}(\operatorname{Sg} S, y)$ and so that $\left(p_{k}\right) \cap \operatorname{Sg} S$ is empty.

5.3. (a) Define sequences $\left(q_{k}\right) \subset C^{N},\left(r_{k}\right) \subset C^{N-1}$ and $\left(s_{k}\right) \subset C$ by $f_{j}^{-1}\left(p_{k}\right)=q_{k}$ $=\left(r_{k}, s_{k}\right)$.

(b) Let $W=\lim _{k} a_{k} f_{j}\left(r_{k}, 0\right)$ and $t=\lim _{k} a_{k} f_{j}\left(0, s_{k}\right)$.

From 4.1(b) we see that $W$ exists. We want to show that $t$ exists and that $v=w+t$. This would complete the proof for $y=0$. As in [2] we see that given $\varepsilon>0$ there is an integer $L$ such that, if $k \geqq L$,

$$
\left|f_{j}\left(q_{k}\right)-f_{j}\left(0, s_{k}\right)-f_{j_{\bullet}}(0)\left(q_{k}-\left(0, s_{k}\right)\right)\right| \leqq \varepsilon\left|q_{k}-\left(0, s_{k}\right)\right| \text {. }
$$

Since $q_{k}-\left(0, s_{k}\right)=\left(r_{k}, 0\right)=f_{j}\left(r_{k}, 0\right)$ we see that $\varepsilon\left|a_{k}\left(q_{k}-\left(0, s_{k}\right)\right)\right|$ is near $\varepsilon|w|$ for $k$ large. From $4.1(\mathrm{~b})$ and 4.6 we see that $f_{j_{*}}(0)\left(q_{k}-\left(0, s_{k}\right)\right)=f_{j}\left(r_{k}, 0\right)$ and so

$$
a_{k}\left[f_{j}\left(q_{k}\right)-f_{j_{*}}(0)\left(q_{k}-\left(0, s_{k}\right)\right)\right]
$$

is near $v-w$ for $k$ large. The above together show that $t$ exists and $t=v-w$. 
If $y \neq 0$ we change coordinates by a translation $\alpha$ with $\alpha(y)=0$; If we set $\tilde{V}=\alpha(V)$ then $\alpha\left(V_{j, y}^{1}\right)=\tilde{V}_{j, 0}^{1}$ and one sees easily that we are reduced to the above case.

5.4. Corollary. For $y \in J, C_{3}(V, y)$ is the union of a finite number of n-planes.

Proof. As shown in [2], $C_{3}(V, y)=\bigcup_{j} C_{3}\left(V_{j, y}, y\right)$.

We want to show that $C_{3}\left(V_{j}\right) \mid S$ is an $n$-plane bundle. To do this we introduce an auxiliary tangent space. Let $y \in S$.

5.5. Definition. (a) $K_{s}\left(V_{j}, y\right)=\left\{v \in C^{m}\right.$ : there are sequences $\left(p_{k}\right) \subset V_{j},\left(t_{k}\right) \subset S$ and $\left(a_{k}\right) \subset C$ such that $\left(p_{k}\right) \rightarrow y, p_{k} \in V_{j, t_{k}}^{\perp}$, and $\left.\left(a_{k}\left(p_{k}-t_{k}\right)\right) \rightarrow v\right\}$.

(b) $K_{s}\left(V_{j}\right)=\bigcup_{y \in S}(y) \times K_{s}\left(V_{j}, y\right)$.

It is shown in [3] that $K_{s}\left(V_{j}\right)$ is an analytic subset of $S \times C^{m}$ of dimension $n$. Using this we see that $K_{s}\left(V_{j}\right)$ is actually a holomorphic family of cones over $S$. 3.1(b) is immediate and 3.1(a) holds since $C_{3}\left(V_{j, y}^{1}, y\right) \subset K_{s}\left(V_{j, y}, y\right)$. Let $f: S \times C^{m}$ $-(0) \rightarrow S \times \boldsymbol{P}^{m-1}$ be as in 3.2 , then $\tilde{K}=f\left(K_{s}\left(V_{j}\right)-S \times(0)\right)$ is an analytic subset of $S \times P^{m-1}$ of dimension $n-1$. Define a function $h: S \rightarrow \widetilde{K}$ as follows:

$$
h(y)=f\left((y) \times C_{3}\left(V_{j, y}^{\perp}, y\right)-(0)\right) \text { for } y \in S .
$$

5.6. LeMma. $h$ is continuous as a map into $S \times \boldsymbol{P}^{m-1}$.

Proof. We show continuity at $x_{0}$, the same proof will work at any other point. Pick $p \in S$, and write $p=\left(p^{\prime}, 0\right)$ where $p^{\prime} \in C_{x_{1}, \ldots, x_{n-1}}$. Let $\left(p_{l}\right) \subset V_{j, p}^{\perp}$ such that $\left(p_{l}\right) \rightarrow p$ and define $\left(s_{l}\right) \subset C$ by $\left(p^{\prime}, s_{l}\right)=f_{j}^{-1}\left(p_{l}\right)$. From $4.5, V_{j}$ is essential so by 4.6 we may write $f_{i, j}=\sum_{k \geqq p_{j}} f_{i, j}^{k} x_{n}^{k}$. If we set $\left(a_{l}\right)=\left(1 /\left|s_{l}\right|^{p_{j}}\right)$ then

$$
\begin{aligned}
\lim _{l \rightarrow \infty}\left|a_{l}\left(p_{l}-p\right)\right|^{2} & =\lim _{l \rightarrow \infty}\left[1+\sum_{i=n+1}^{m}\left|\sum_{k \geqq p_{j}} f_{i, j}^{k}(p) s_{l}^{j-p_{j}}\right|^{2}\right] \\
& =1+\sum_{i=n+1}^{m}\left|f_{i, j}^{p_{j}}(p)\right|^{2} .
\end{aligned}
$$

Let us call this quantity $A$. Now we have

$$
\begin{aligned}
\left(\left|p_{l}-p, f_{j}\left(0, s_{l}\right)\right|^{*}\right)^{2} & \leqq \frac{\left|a_{l}\left(p_{l}-p\right)-a_{l} f_{j}\left(0, s_{l}\right)\right|^{2}}{\left|a_{l}\left(p_{l}-p\right)\right|^{2}} \\
& \leqq \frac{\sum_{i=n+1}^{m}\left|\sum_{k \geqq p_{j}}\left(f_{i, j}^{k}(p)-f_{i, j}^{k}(0)\right) s_{l}^{k-p_{j}}\right|^{2}}{\left|a_{l}\left(p_{l}-p\right)\right|^{2}}
\end{aligned}
$$

The limit of the right side as $l \rightarrow \infty$ is $1 / A\left(\sum_{i=n+1}^{m}\left|f_{i, j}^{p}(p)-f_{i, j}^{p_{j}(0)}\right|^{2}\right)$. Thus for $|p|$ small and $l$ large $\left|p_{l}-p, f_{j}\left(0, s_{l}\right)\right|^{*}$ can be made arbitrarily small. Let $v_{1} \in C_{3}\left(V_{j, p}^{1}, p\right)$ and $v_{2} \in C_{3}\left(V_{j, 0}^{\perp}, 0\right)$, both nonzero. It follows from Lemma 8.15 in [3] that for $l$ sufficiently large both $\left|v_{1}, p_{l}-p\right|^{*}$ and $\left|v_{2}, f_{j}\left(0, s_{l}\right)\right|^{*}$ will be arbitrarily small. Combining the above we have that, for $|p|$ small, $\left|v_{1}, v_{2}\right|^{*}$ can be made arbitrarily small. This completes the proof.

\subsection{LeMmA. For $p \in S, K_{s}\left(V_{j, p}\right)=C_{3}\left(V_{j, p}^{1}, p\right)$.}


Proof. Let $\left(P_{l}\right),\left(t_{l}\right),\left(a_{l}\right)$ and $v$ be as in 5.5(a). Define $\left(s_{l}\right) \subset C$ by $\left(r_{l}, s_{l}\right)=f_{j}^{-1}\left(p_{l}\right)$ where $t_{l}=\left(r_{l}, 0\right)$. This is as in 5.3. We have from 4.6

$$
a_{l}\left(p_{l}-t_{l}\right)=a_{l}\left(s_{l}^{p_{j}}, \sum_{k \geqq p_{j}} f_{n+1, j}^{k}\left(r_{l}\right) s_{l}^{k}, \ldots, \sum_{k \geqq p_{j}} f_{m, j}^{k}\left(r_{l}\right) p_{l}^{k}\right)
$$

and so $\alpha=\lim _{l \rightarrow \infty} a_{l} s^{p_{j}}$ must exist. Then

$$
\lim _{l \rightarrow \infty} a_{l}\left(p_{l}-t_{l}\right)=\alpha\left(1, f_{n+1, j}^{p_{j}}\left(p^{\prime}\right), \ldots, f_{m, j}^{p_{j}}\left(p^{\prime}\right)\right)
$$

when $p=\left(p^{\prime}, 0\right)$. Thus $K_{s}\left(V_{j}, p\right)$ is a line. Since $C_{3}\left(V_{j, p}^{1}, p\right) \subset K_{s}\left(V_{j}, p\right)$ we are done.

5.8. Proposition. $C_{3}\left(V_{j}\right) \mid S$ is an n-plane bundle over $S$.

Proof. Let $\tilde{g}: \tilde{K} \rightarrow \tilde{S}$ be the restriction of the projection $S \times P^{m-1} \rightarrow S$. From 5.7 it follows that $\tilde{g}$ is a homeomorphism, since $g^{-1}=h$, and so $\tilde{K}$ is globally irreducible. Now from 1.7 we see there is a proper analytic subset $A \subset \tilde{K}$ so that $\tilde{g} \mid \tilde{K}-A \rightarrow S$ $-\tilde{g}(A)$ is biholomorphic. By the Riemann extension theorem $h$ extends biholomorphically from $S-\tilde{g}(A)$ to $S$ and $\tilde{K}$ is a manifold. Taking local coordinates in $\boldsymbol{P}^{m-1}$ and using the definition of $f$ we see that $K_{s}\left(V_{j}\right)$ is a line bundle. Now the proposition follows from 5.2 .

We next show that $C_{3}\left(V_{j}\right)\left|S=C_{4}\left(V_{j}\right)\right| S$. Since $C_{i}(V, y)=\bigcup_{j} C_{i}\left(V_{j}, y\right)$ for $y \in S$ it follows from 3.9 in [3] that the closure of $C_{3}(V) \mid J$ in $\mathrm{Sg} V \times C^{m}$ is an analytic set. This set will in general be larger than $C_{3}(V) \mid \mathrm{Sg} V$. See for example 7.8 in [2].

5.9. Proposition. $C_{3}\left(V_{j}, y\right)=C_{4}\left(V_{j}, y\right)$ for $y \in S$.

Proof. We will show that $C_{4}\left(V_{j}, y\right)$ is an $n$-plane. Since $C_{3}\left(V_{j}, y\right) \subset C_{4}\left(V_{j}, y\right)$ and is also $n$-dimensional this is sufficient. Let $v \in C_{4}\left(V_{j}, j\right)$ and pick $\left(p_{l}\right) \subset V_{j}-S$ and $\left(v_{l}\right) \subset C^{m}$ with $\left(p_{j}\right) \rightarrow p, v_{l} \in T\left(V_{j}, p_{l}\right)$, and $\left(v_{i}\right) \rightarrow v$. Define $\left(q_{l}\right),\left(r_{l}\right),\left(s_{l}\right)$ as in 5.3 and $\left(\tilde{v}_{l}\right) \subset C^{n}$ by $\tilde{v}_{l}=f_{j_{0}}^{-1}\left(v_{l}\right)$. Write $\tilde{v}_{l}=\left(\tilde{v}_{l}^{1}, \ldots, \tilde{v}_{l}^{n}\right)$. For $x \in \tilde{D}$ we can write $J f_{j}(x)$, the Jacobian of $f_{j}$ at $x$, in block form as follows:

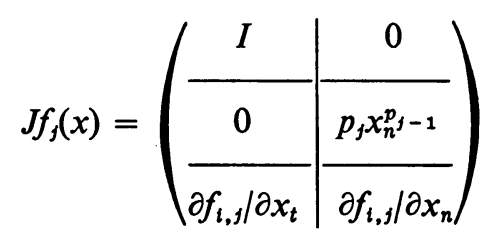

Here $I$ is the $n-1 \times n-1$ identity matrix. From this we see that the first $n-1$ components of $v_{l}$ are $\tilde{v}_{l}^{1}, \ldots, \tilde{v}_{l}^{n-1}$ and so the sequences $\left(\tilde{v}_{l}^{t}\right)$ must converge for $i=1, \ldots, n-1$. The $n$th component is $\tilde{v}_{l}^{n} \cdot p_{j} s_{l}^{p_{j-1}}$ and so $\left(\tilde{v}_{l}^{n} \cdot s_{l}^{p_{j-1}}\right)$ converges. From 4.6 we see that $s p_{j}$ divides $\partial f_{i, j}\left(q_{l}\right) / \partial x_{t}$ for $t \leqq n-1$ and so $\lim _{l \rightarrow \infty} \tilde{v}_{l}^{t} \cdot \partial f_{i, j}\left(q_{l}\right) / \partial x_{t}=0$ for $t \leqq n-1$. Thus the components of $v$ are given by

$$
\begin{array}{ll}
v_{i}=\lim _{l \rightarrow \infty} \tilde{v}_{l}^{t} & \text { for } i \leqq n-1, \\
v_{n}=p_{j} \cdot \lim _{l \rightarrow \infty} \tilde{v}_{l}^{n} \cdot s_{l}^{p^{j-1}}, & \\
v_{i}=p_{j} f_{i, j}^{p_{j}}\left(p^{\prime}\right) \cdot \lim _{l \rightarrow \infty} \tilde{v}_{l}^{n} \cdot s_{l}^{p_{j-1}} & \text { for } i>n .
\end{array}
$$

Here $p=\left(p^{\prime}, 0\right)$ as usual. The above equations define a plane so we are done. 
6. Smooth attaching along $J$. Before going to the definition of smooth attaching we need to introduce a bit more machinery. Let $G^{m, n}$ be the grassmannian of $n$-planes in $C^{m}$ through 0 , and let $\widetilde{G}^{m-1, n-1}$ be the associated projective grassmannian. For $K, L \in G^{m, n}$ we have

6.1. Definition. $|K, L|_{n}^{*}=\sup _{u \in K} \inf _{v \in L}|u, v|^{*}$.

The reader is referred to [3] for the properties of $|,|_{n}^{*}$. Let $W \subset V$ be a submanifold of dimension $n-1$, and $y \in W$.

6.2. Definition. We will say that $V$ attaches smoothly to $W$ near $y$ if we can find coordinates $\left(x_{i}\right)$ for $C^{m}$ and a neighborhood $N$ of $y$ so that

(a) $W \cap N=\left\{x \in N: x_{i}=0\right.$ for $\left.i \geqq n\right\}$.

(b) We have a special branched covering $(N, \pi, D)$, defined by the $m-n$ plane $H=\left\{x \in C^{m}: x_{i}=0\right.$ for $\left.i \leqq n\right\}$, with $B(N, \pi)=W \cap N$.

(c) Let $V_{j}$ be the global branches of $N_{y}$. Then $V_{j} \cap W=N \cap W$ and for each $j$ there is a holomorphic function $\alpha^{j}: V_{j} \cap W \rightarrow C_{x_{n}, \ldots, x_{m}}$ such that if $\rho: C^{m} \rightarrow C_{x_{n}, \ldots, x_{m}}$ is the coordinate projection then for $z \in W$

$$
C_{3}\left(V_{j}, z\right)=\left\{v \in C^{m}: \rho(v)=a \alpha^{j}(z) \text { for } a \in C\right\} .
$$

(d) For $x \in V_{j}-W$ let $T(x)$ be $C_{x_{1}, \ldots, x_{n-1}}$ cross the line spanned by $\rho(x)$. Then given $\varepsilon>0$ there is $\delta>0$ such that if $|\rho(x)|<\delta$ then $\left|\rho(x), \alpha^{j}(\tilde{x})\right|^{*}<\varepsilon$ and

$$
\left|T(x), T\left(V_{j}, x\right)\right|_{m}^{*}<\varepsilon \text {. }
$$

Here $\tilde{x}$ is defined by $x=(\tilde{x}, \rho(x))$.

Set $W_{1}=W \cap \operatorname{Sg} V$ and define $W_{1}^{(k)}$ as in 3.2. Let

$$
W_{2}=[W \cap(\operatorname{Sg} V-J)] \cup\left[\bigcup_{k<n-1} W_{1}^{(k)}\right]
$$

\subsection{Proposition. If $y \in W-W_{2}$ then $V$ attaches smoothly to $W$ near $y$.}

Proof. The proposition is trivial if $y \in V-\operatorname{Sg} V$ so suppose $y \in \operatorname{Sg} V$. From the definition of $W_{2}$ we see that $W=\operatorname{Sg} V$ near $y$ and $y \in J$. We can take $(N, \pi, D)$ as in the preceding parts of this section so (a) and (b) hold. To conform to our previous usage in $\S 5$, we will write $S$ for $W \cap N$. From the proof of 5.8 we see that, for each $V_{j}, K_{s}\left(V_{j}\right)$ is a sub-line bundle of $S \times C_{x_{n}, \ldots, x_{m}}$. By shrinking $D$ we can take $\alpha^{j}$ to be sections of these bundles. Since $K_{s}\left(V_{j}, y\right)=C_{3}\left(V_{j}^{\perp}, y\right)$ and $H$ is transverse to $C_{3}\left(V_{j}^{\perp}, y\right)$ we see that the first component of $\alpha^{j}$ cannot vanish and so we can normalize $\alpha^{j}$ so that its just component is identically 1. (c) now follows from 5.2.

We may replace $N$ with an open subset $N^{\prime}$ with $y \in N^{\prime}$ and $N^{\prime} \subset \bar{N}$. If the first condition in (d) did not hold on $N^{\prime}$ then we could find $\varepsilon>0$ and $x \in N$ such that there is a sequence $\left(x_{l}\right) \subset V_{j}-S$ for some $j$ with $\left(x_{l}\right) \rightarrow x$ and $\left|\rho\left(x_{l}\right), \alpha^{j}\left(\tilde{x}_{l}\right)\right|^{*} \geqq \varepsilon$. Since $\alpha^{j}$ is continuous and $\alpha^{j}(\tilde{x})=K_{x}\left(V_{j}, \tilde{x}\right)$ this is a contradiction. For the second condition note that $\left|C_{4}\left(V_{j}, \tilde{x}\right), T\left(V_{j}, x\right)\right|_{n}^{*}<\varepsilon / 2$ for $|\rho(x)|$ sufficiently small. This follows easily by contradiction from all the definitions of $C_{4}(V, \tilde{x})$. On the other hand one sees from the argument above that

$$
\left|T(x), C_{x_{1}, \ldots, x_{n-1}} \times K_{s}\left(V_{j}, \tilde{x}\right)\right|_{n}^{*}<\varepsilon / 2 \text { for }|\rho(x)| \text { small. }
$$

Since $|,|_{n}^{*}$ satisfies the "triangle inequality" this completes the proof. 
In [3] Whitney proved a result similar to 6.3 (Proposition 14.4). However his proof did not show that the set of points $G \subset W$ near which $V$ did not attach smoothly to $W$ was an analytic subset of $W$. Whitney conjectured that this was so and that $G$ would be defined intrinsically by $V$ and $W$. Our proof shows that $G$ is at least contained in such a set.

6.4. Definition. Let $V, W$ be as above. We say that $V$ is regular over $W$ if for $x \in W$

(a) If there is $\left(p_{j}\right) \subset V-\operatorname{Sg} V$ with $\left(p_{j}\right) \rightarrow x$ and $T\left(V, p_{j}\right) \rightarrow T$ then $T(W, x) \subset T$.

(b) If there are $\left(p_{j}\right) \subset V-\operatorname{Sg} V,\left(t_{j}\right) \subset W,\left(a_{j}\right) \subset C$ with $\left(p_{j}\right) \rightarrow x,\left(t_{j}\right) \rightarrow x$, $\left(T\left(V, p_{j}\right)\right) \rightarrow T$ and $a_{j}\left(p_{j}-t_{j}\right) \rightarrow v$ then $v \in T$.

It follows easily from the proof of 6.3 (condition (d)) that $V$ is regular over $W-W_{2}$. In particular if $\operatorname{dim} \operatorname{Sg} V=n-1$ then $V-\operatorname{Sg} V$ and $J$ form the first two strata in a regular stratification of $V$. (See [3] for details on stratification.) By using Whitney's technique of "cutting down dimensions" one can show that if $W$ is a submanifold of dimension $s$ then each $x \in W$ has a neighborhood $N$ which contains an analytic set $\bar{V}$ with $\operatorname{dim} \bar{V} \equiv s+1$ such that $\bar{V}$ attaches smoothly to $W$ on the complement of an analytic subset $\bar{W}$ of $W$. Using this one can modify the proof of Lemma 13.5 in [3] to show the existence of "smooth wings" from $W-\bar{W}$ into $V$. As in $[3,13.5]$ we may avoid a given analytic subset of $V$. The details of this argument are left to the reader.

7. Normal pseudoflatness and semi-analytic fibration. We continue the notation introduced in $\$ \S 4$ and 5 . We wish to obtain an alternative formulation of the fact that $K_{s}\left(V_{j}\right)$ is a line bundle. To do this we introduce the notion of a blow up.

Given $x \in C^{m}$ we can write it as $x=(\alpha(x), \beta(x))$ with $\alpha(x) \in C_{x_{1}, \ldots, x_{n-1}}$ and $\beta(x) \in C_{x_{n}, \ldots, x_{m}}$. Let $\boldsymbol{P}^{m-n-1}$ be the projective space defined by $\boldsymbol{C}_{x_{n}, \ldots, x_{m}}$ and $\rho: C_{x_{n}, \ldots, x_{m}}-(0) \rightarrow \boldsymbol{P}^{m-n-1}$ the canonical projection.

7.1. Definition. (a) $B l=\left\{(x, v) \in C^{m} \times \boldsymbol{P}^{m-n-1}\right.$ : for $v^{\prime} \in \rho^{-1}(v)$, rank $\left.\left({ }^{x-\alpha(x)} v^{\prime}\right)<2\right\}$.

(b) $B l^{*}$ is the closure of $B l-\left\{x \in C^{m}: \beta(x)=0\right\} \times \boldsymbol{P}^{m-n-1}$ in $\boldsymbol{C}^{m} \times \boldsymbol{P}^{m-n-1}$.

(c) $V_{j}^{*}=B l^{*} \cap\left(V_{j} \times \boldsymbol{P}^{m-n-1}\right)$ for each $j$.

As observed in [3], $B l$ and $B l^{*}$ are analytic subsets of $C^{m} \times P^{m-n-1}$ and so if we write $N=V \cap U$, for $U$ open in $C^{m}$, we have $V_{j}^{*}$ an analytic subset of $U \times \boldsymbol{P}^{m-n-1}$. $V_{j}^{*}$ is the closure of $\left\{(x, v) \in V_{j} \times \boldsymbol{P}^{m-n-1}: \beta(x) \neq 0\right.$ and $\left.(\rho \cdot \beta)(x)=v\right\}$. From this we see that

7.2. $\rho^{-1}\left(V_{j}^{*} \cap\left\{x \in C^{m}: \beta(x)=0\right\} \times P^{m-n-1}\right)=\left[K_{s}\left(V_{j}\right)-(0)\right] \times C_{x_{1}, \ldots, x_{n-1}}$. For $x \in S,(x) \times K_{s}\left(V_{j}, x\right)$ is the normal cone of $s$ in $V_{j}$ at $x$, as defined in [11].

(This depends upon an identification of the above with Hironaka's method of blowing up. We will not go into this here.) It now follows from $\$ 5$ that

7.3. Proposition. The normal cone of $s$ in $V_{j}$ is a line bundle for each $j$.

We may form $V^{*}$ as above. It is easy to see that $V^{*}=\bigcup_{j} V_{j}^{*}$, and so the normal cone of $s$ in $V$ at $x$ is $(x) \times K_{s}(V, x)$. Thus for each $x \in S$, the normal cone of $s$ in $V$ 
at $x$ is of constant dimension. This constancy of dimension is what Hironaka calls normal pseudoflatness of $V$ along $s$. Since $J$ can be taken to be the stratum of codimension 1 in a regular stratification of $V$ the above is a special case of the main result of [11]. There it is shown that given a regular stratification of an analytic set, the set is normally pseudoflat along each stratum.

In order to relate the above to the notion of normal-flatness introduced in [13] we have the following definition.

7.4. Definition. Let $X, Y$ be analytic sets and $f: X \rightarrow Y$ be a holomorphic map. $f$ is said to be flat if, for any $y \in Y$ and $x \in f^{-1}(y)$, the map $f^{*}: \mathcal{O}_{Y, y} \rightarrow \mathcal{O}_{X, x}$ defines a flat $\mathcal{O}_{Y, y}$-module.

7.5. Proposition. The map $f: K_{s}(V) \rightarrow S$, given by restriction of the projection $S \times C_{x_{n}, \ldots, x_{m}} \rightarrow S$, is flat.

Proof. Since $K_{s}(V)$ is a holomorphic family of cones, it determines an analytic set $K \subset S \times \boldsymbol{P}^{m-n-1}$. (See the remarks following 5.5.) Projection on $S$ induces a map $f: K \rightarrow S$ which represents $K$ as a branched cover of $S$. As in $\S 5$ we see that $K_{s}(V)$ is isomorphic to $K \times C$ in such a way that if $\pi: K_{s}(V) \rightarrow K$ is given by projection, we have $f=f \circ \pi$. By Theorem 2 in [10] the map $\pi$ is flat. For $y \in S$, and $x \in f^{-1}(y)$, set $v(x)=$ dimension of $C \otimes \mathcal{O}_{s, y} \mathcal{O}_{K, x}$ and $v(y)=\sum_{x \in \mathcal{Y}^{-1}(y)} v(x)$. From 5.6 and 5.8 one knows that if $\left(K_{j}\right)$ are the global branches of $K$ then $f^{\prime} \mid K_{j}$ is biholomorphic. One sees easily that this implies $v$ is constant (and equals the number of branches). By Theorem 1 in [10] this implies that $f$ is flat, and so $f$ is also flat.

We will conclude this section with a result that describes the topology of $V$ near a point of $J$.

Let $H$ be as in 4.3(a). We may assume that $U=S \times U$, where $U_{1}=U \cap H$.

7.6. Definition. A map $\varphi: U \rightarrow U$ will be called a semi-analytic fibration for $N$ if

(a) $\varphi$ is a homeomorphism;

(b) $\varphi \mid(x) \times U_{1}$ is biholomorphic for all $x \in S$;

(c) $\varphi \mid(0) \times U_{1}$ and $\varphi \mid S \times(0)$ are just the inclusion maps;

(d) for $y \in U_{1}$, if $\varphi(S \times(y)) \cap N$ is not empty then $\varphi(S \times(y)) \subset N$.

7.7. Proposition. There is a semi-analytic fibration for $N$.

Proof. Let $U^{i}=U \cap C_{x_{1}, \ldots, x_{i}, x_{i}}$ for $i=n+1, \ldots, m$, and let $\left(\pi^{i}\right),\left(\pi_{i}\right)$ and $\pi$ denote the extensions of the maps defined in 2.6 and 2.7 to the appropriate ambient spaces. We note that the hypotheses of Theorem 12.1 in [2] are only used to obtain branched covers with the same properties as the $\left(V^{i}, \pi_{i}, D\right)$. Thus for each $i$ we have a semi-analytic fibration $\varphi_{i}: U^{i} \rightarrow U^{i}$ with $\pi_{i} \circ \varphi_{i}: U_{i} \rightarrow D$ given by projection on the first $n$-coordinates. Because of this compatibility the $\varphi_{i}$ define a map $\varphi: U \rightarrow U$. That 7.6(a), (b) and (c) hold for $\varphi$ is immediate, from the fact that they hold for each $i$. Since $\pi^{i}: V \rightarrow V^{i}$ is a homeomorphism (d) also holds, so we are done.

7.8. Corollary. Let $V_{0}^{\frac{1}{1}}=H \cap V . \varphi\left(S \times V_{0}^{1}\right)=V$ and so $V$ is topologically a product. 
Proof. From 7.6(c) we have $\varphi\left((0) \times V_{0}^{1}\right)=\left((0) \times V_{0}^{1}\right)$. Let $y \in V_{0}^{1}$ then $(0, y)$ $\in \varphi(S \times(y)) \cap V$ and so $\varphi(S \times(y)) \subset V$. Since this is true for all $y, \varphi\left(S \times V_{0}^{1}\right) \subset V$. On the other hand for $x \in\left\{x \in D: x_{n}=0\right\}$ then $(\pi \circ \varphi)^{-1}(x) \cap\left(S \times V_{0}^{1}\right)$ and $\pi^{-1}(x) \cap V$ both contain precisely $p$ distinct points and so $\varphi\left(S \times V_{0}^{\perp}-(0)\right)=V-S$. Combining this with $7.6(\mathrm{c})$ completes the proof.

\section{BIBLIOGRAPHY}

1. R. C. Gunning and H. Rossi, Analytic functions of several complex variables, PrenticeHall, Englewood Cliffs, N. J., 1965. MR 31 \#4927.

2. H. Whitney, Local properties of analytic varieties, Differential and Combinatorial Topology (A Symposium in Honor of Marston Morse), Princeton Univ. Press, Princeton, N. J., 1965. MR $32 \# 5924$.

3. - Tangents to an anlytic variety, Ann. of Math. (2) 81 (1965), 496-549. MR 33 \#745.

4. R. Draper, Intersection theory in analytic geometry, Math. Ann. 180 (1969), 175-204. MR 40 \#403.

5. R. Narasimhan, Introduction to the theory of analytic spaces, Lecture Notes in Math., no. 25, Springer-Verlag, Berlin, 1966. MR 36 \#428.

6. S. Abhyankar, Local analytic geometry, Pure and Appl. Math., vol. 14, Academic Press, New York, 1964. MR 31 \#173.

7. O. Zariski, Equisingular points on algebraic varieties, Seminari 1962/63 Anal. Alg. Geom. e Topol., vol. 1, Ist. Naz. Alta Mat., Ediz. Cremonese, Rome, 1965, pp. 164-177. MR 33 \#7336.

8. I. Kimura, On normal analytic sets. I, II, Proc. Japan Acad. 43 (1967), 464-468; ibid. 43 (1967), 719-722. MR 37 \#1649.

9. M. Hervé, Math. Reviews 37 (1968), 309 (article \#1649).

10. A. Douady, Flatness and privilege, Enseignement Math. (2) 14 (1968), 47-74. MR 38 \#4716.

11. H. Hironaka, Normal cones in analytic Whitney stratifications, Inst. Hautes Études Sci. Publ. Math., no. 31, 1970.

12. N. Kuhlmann, Algebraic function fields on complex analytic spaces, Proc. Conf. Complex Analysis (Minneapolis, 1964), Springer, Berlin, 1965, pp. 155-172. MR 30 \#4977.

13. H. Hironaka, Resolution of singularities of algebraic varieties over fields of characteristic zero. I, II, Ann. of Math. (2) 79 (1964), 109-203; 205-326. MR 33 \#7333.

14. J. Stutz, Equisingularity and equisaturation in codimension 1, preprint.

Department of Mathematics, Massachusetts institute of Technology, Cambridge, MASSACHUSETTS 02139

Current address: Department of Mathematics, State University of New York at Albany, Albany, New York 12203 